\title{
Erratum to: Comprehensive Study of Metamorphic Core Complex in Southern Liaodong Peninsula, NE China
}

Zhong Mishan ${ }^{1 *}$, Wu Zijie ${ }^{1}$, Liang Shuai ${ }^{2}$, Zhang Guoren ${ }^{1}$, Wang Qi $^{1}$, Gao Fuliang ${ }^{1}$, Pan Yuqi ${ }^{1}$, Gao Yongzhao ${ }^{1}$ and Fan Shuo ${ }^{1}$

${ }^{1}$ Liaoning Provincial Institute of Geological Exploration Co., Ltd,116100 Dalian, Liaoning, China

${ }^{2}$ School of Earth Science and Engineering, Sun Yat-Sen University, 510275 Guangzhou, Guangdong, China

Original article: E3S Web of Conferences 131, 01032 (2019), https://doi.org/10.1051/e3sconf/201913101032

The author list and the authors' information should be the following:

Zhong Mishan ${ }^{1 *}$, Wu Zijie ${ }^{1}$, Liang Shuai ${ }^{2}$, Zhang Guoren ${ }^{1}$, Wang Qi $^{1}$, Gao Fuliang ${ }^{1}$, Pan Yuqi ${ }^{1}$, Gao Yongzhao ${ }^{1}$ and Fan Shuo $^{1}$

${ }^{1}$ Liaoning Provincial Institute of Geological Exploration Co., Ltd,116100 Dalian, Liaoning, China

${ }^{2}$ School of Earth Science and Engineering, Sun Yat-Sen University, 510275 Guangzhou, Guangdong, China 